\title{
Erratum: Constructing Multiclass Learners from Binary Learners: A Simple Black-Box Analysis of the Generalization Errors
}

\author{
Jittat Fakcharoenphol ${ }^{1}$ and Boonserm Kijsirikul ${ }^{2}$ \\ 1 Department of Computer Engineering, Kasetsart University, Bangkok, Thailand. \\ jtf@ku.ac.th \\ 2 Department of Computer Engineering, Chulalongkorn University, Bangkok, Thailand. \\ Boonserm.K@chula.ac.th
}

\begin{abstract}
There are errors in our paper "Constructing Multiclass Learners from Binary Learners: A Simple Black-Box Analysis of the Generalization Errors," which appeared in ALT'05 [3]. The errors are related to our uses of union bounds. We briefly describe the problem and discuss which of our results can be shown to hold. We also provide a counter example for our previous claim.
\end{abstract}

\section{Background}

In [3], we analyzed various multiclass learning algorithms that use binary classifications as subroutines. We viewed binary classifiers as black-boxes and analyzed the error rate of the multiclass construction as a function of binary error rates. This approach is mainly known as learning reductions [1].

In what follows, we assume that the readers are familiar with the pair-wise reductions such as Decision Directed Acyclic Graphs [5] (DDAG), Adaptive Directed Acyclic Graphs [4] (ADAG).

\section{The errors}

The problems in that paper in our analysis of adaptive constructions, i.e., those whose set of invoked binary classifiers changes over the input. They include DDAG, ADAG, and Randomized Decision Directed Acyclic Graphs (R-DDAG). More specifically, the errors are regarding our use of the union bound.

To see the problem, we consider our analysis of ADAG for the problem with $k$ classes. We start with the setting. Let $D$ be the distribution over $X$, set of all data points. There are $\left(\begin{array}{c}k \\ 2\end{array}\right)$ classifiers: there is a binary classifier $A_{i, j}$ for each pair $i \neq j$. Error rate of classifier $A_{i, j}, \epsilon_{i, j}$ is defined to be

$$
\operatorname{Pr}_{x \sim D}\left[A_{i, j}(x) \text { gives a wrong prediction } \mid x \text { belongs to class } i \text { or class } j\right] .
$$

In the ADAG reduction, we have a full binary tree $T$, with $k$ leaves initially labelled with all classes, while all internal nodes are unlabelled. Call the leaf labelled with $i, L_{i}$. Given a data point $x$, the prediction algorithm picks any unlabelled node $u$ whose children are all labelled. Suppose that they are labelled with $i$ and $j$. We then call $A_{i, j}(x)$ and assign the result of the classifier as the label of $u$. The algorithm iterates until there is no unlabelled node left. The multiclass prediction is the label of the root node.

Our analysis first assume that the data point belongs to class $i$. Let $\mathcal{I}(i)$ denote the set of internal nodes of $T$ on the path from $L_{i}$ to the root. The algorithm makes a wrong prediction if any classifier called on these nodes make mistakes. Denote by $\mathcal{E}(u)$ the event that the classifier on node $u$ makes a wrong prediction; thus, the multiclass error rate is $\operatorname{Pr}\left[\bigcup_{u \in \mathcal{I}(i)} \mathcal{E}(u)\right] \leq \sum_{u \in \mathcal{I}(i)} \operatorname{Pr}[\mathcal{E}(u)]$.

For any node $u$, let $\mathcal{L}(i)$ denote the set of leaf labels in the subtree rooted at $u$. We claim, erroneously, that $\max _{j \in \mathcal{L}(u)} \epsilon_{i, j}$ is an upperbound on $\operatorname{Pr}[\mathcal{E}(u)]$.

If this were true, we would have that, since $|I(i)| \leq\lceil\log k\rceil$, the error rate is at most $\sum_{j=1}^{\lceil\log k\rceil} \epsilon_{i, r_{j}}$, when $r_{j}$ is the class $c$ with the $j$-th largest error rate $\epsilon_{i, c}$. 
The above claim would have work if each classifiers $A_{i, j}$ is randomized and for any data point $x$, it makes a mistake with probability $\epsilon_{i, j}$. However, usually for a fixed $x$, the error is not random.

The correct analysis of $\operatorname{Pr}[\mathcal{E}(u)]$ must consider all binary classifiers $A_{i, j}$ for $j \in \mathcal{L}(u)$. Let event $\mathcal{F}(i, j)$ denote the event that the classifier $A_{i, j}$ is used at node $u$ and makes a wrong prediction. Thus, $\operatorname{Pr}[\mathcal{E}(u)]=$ $\operatorname{Pr}\left[\bigcup_{j \in \mathcal{L}(u)} \mathcal{F}(i, j)\right]=\sum_{j \in \mathcal{L}(u)} \operatorname{Pr}[\mathcal{F}(i, j)]$. With no further assumption, we can only bound this with $\sum_{j \in \mathcal{L}(u)} \epsilon_{i, j}$, using the union bound. Thus, the probability of making mistake is at most $\sum_{j \neq i} \epsilon_{i, j}$, using again the union bound. In the case of uniform error rate, this only gives the bound of $(k-1) \epsilon$. This analysis is tight (see an example in Section 3).

The erroneous theorems are Theorems 2, 3, and 4 . In Theorem 2, we claim an upper bound $\delta_{i}$ for input from class $i$ to be at most $\max \left\{\sum_{j<i} \epsilon_{i, j}, \sum_{j>i} \epsilon_{i, j}\right\}$. The correct upperbound is $\sum_{j \neq i} \epsilon_{i, j}$. This is the correct bounds for Theorems 3 and 4 as well.

Our analyses of non-adaptive constructions (Theorems 1, 5, and 6) remain correct.

\section{A tight example}

For any $k>2$. We first describe the probability space for the input. Let $[k]=\{1, \ldots, k\}$. The sample space $\Omega$ is $\{(a, b): a \in[k], b \in[k]-\{a\}\}$. The correct multiclass prediction for $x=(a, b)$ is $f(x)=a$.

We now define each binary classifier $A_{i, j}$. Given $x=(a, b), A_{i, j}$ proceeds as follows.

1. if $a \in\{i, j\}$, it correctly predicts $a$ iff $b \notin\{i, j\}$, otherwise it incorrectly predicts $c \in\{i, j\}-a$.

2. if $a \notin\{i, j\}$ but $b \in\{i, j\}$, it predicts $b$.

3. otherwise, it predicts either $i$ or $j$ arbitrary.

Note that for classifier $A_{i, j}$ and a uniformly random $x=(a, b) \in \Omega$ from class $i$ or $j, A_{i, j}$ predicts incorrectly only when $b \in\{i, j\}-\{a\}$, which occurs with probability $1 /(k-1)$. That is,

$$
\epsilon_{i, j}=\operatorname{Pr}\left[A_{i, j} \text { predicts incorrectly } \mid x \text { is from class } i \text { or } j\right]=1 /(k-1) .
$$

However, note that when the multiclass classifier (either constructed using DDAG, ADAG, or RDDAG) is given $x=(a, b)$, the construction ensures that $A_{a, b}$ is on the prediction path. Clearly $A_{a, b}$ always give a wrong prediction for $(a, b)$. Therefore, the multiclass error rate is 1 , while the binary error rate is only $1 /(k-1)$.

This example also shows that ADAG cannot transform regrets efficiently, if we define a binary regret for a classifier $A_{i, j}$ to be

$$
\operatorname{Pr}_{x \sim D}\left[A_{i, j}(x) \text { is wrong } \mid x \text { from } i \text { or } j\right]-\operatorname{Pr}_{x \sim D}\left[A_{i, j}^{*}(x) \text { is wrong } \mid x \text { from } i \text { or } j\right],
$$

where $A_{i, j}^{*}$ is the bayes optimal classifier. Note that the optimal bayes classifier for this problem makes no errors; thus, the binary errors equal to the binary regret.

\subsection{A modified definition of binary error rate}

From the example, although each binary classifier makes wrong prediction in only $1 /(k-1)$ fraction of the input, it occurs when the classifier is always on the prediction path (again in $1 /(k-1)$ of the time). To capture this into the binary error rate, we can redefine $\hat{\epsilon}_{i, j}$ to be the error conditioned on the event that $A_{i, j}$ lies on the prediction path. More specifically, $\hat{\epsilon}_{i, j}$ is the probability that $A_{i, j}$ predicted incorrectly when the input is from class $i$ or $j$ and $A_{i, j}$ is invoked in the multiclass algorithm.

Given the modified definition, we are able to prove the similar error bounds as previously claimed. (See the full erratum.) However, the error rate $\hat{\epsilon}_{i, j}$ depends heavily on the constructed multiclass classifier. More over, a classifier with small $\epsilon_{i, j}$ might have large $\hat{\epsilon}_{i, j}$. Thus, this conditioning makes the task of training $A_{i, j}$ to minimize $\hat{\epsilon}_{i, j}$ difficult.

This modified definition coincides with the one that Beygelzimer, Langford, and Ravikumar [2] have already used in their recent work on the filter trees. More over, they have provided the solution to the 
training problem we mentioned, as the conditional training algorithm for the filter trees can be seen as an explicit method to train the binary classifier under the new definition of error rates. Our example above supports their belief that the conditioning step is crucial.

\section{Acknowledgement}

We would like to thank John Langford for a useful discussion.

\section{References}

1. A. Beygelzimer, V. Dani, T. Hayes, J. Langford, and B. Zadrozny. Error limiting reductions between classification tasks. In ICML '05: Proceedings of the 22nd international conference on Machine learning, pages 49-56, New York, NY, USA, 2005. ACM.

2. A. Beygelzimer, J. Langford, and P. Ravikumar. Multiclass classification with filter trees. Manuscript. available at URL: http://hunch.net/ j1/projects/reductions/mc_to_b/invertedTree.pdf, 2007.

3. J. Fakcharoenphol and B. Kijsirikul. Constructing multiclass learners from binary learners: A simple black-box analysis of the generalization errors. In ALT, pages 135-147, 2005.

4. B. Kijsirikul, N. Ussivakul, and S. Meknavin. Adaptive directed acyclic graphs for multiclass classification. In PRICAI 2002, pages 158-168, 2002.

5. J. Platt, N. Cristianini, and J. Shawe-Taylor. Large margin DAGs for multiclass classification. In Advance in Neural Information Processing System, volume 12. MIT Press, 2000. 